\title{
TECHNICAL ASPECTS OF UPGRADING COMPOSTING TO ANAEROBIC DIGESTION AND POST-COMPOSTING
}

\author{
MARCO RAGAZZI \& MARCO SCHIAVON \\ Department of Civil Environmental and Mechanical Engineering, University of Trento, Italy
}

\begin{abstract}
The present article analyses the advantages of converting direct composting of the organic fraction of municipal solid waste into a plant with an initial step of anaerobic digestion, Clear advantages come from the energy balance thanks to the role of biogas. Important advantages are also the reduction of surface required for treating the same amount of waste and the positive role of the anaerobic digester for the management of odorigenous compounds. In terms of timing, anaerobic digestion allows decreasing the time spent for the treatment. The saving of surface can be completed by the adoption of a biotrickling filter to replace conventional biofiltration.

Keywords: anaerobic digestion, biofilter, biogas, biotrickling filter, composting, energy, OFMSW, waste.
\end{abstract}

\section{INTRODUCTION}

The amount of produced municipal solid waste (MSW) has undergone a continuous growth in the last decades even in industrialised regions like Europe. Meanwhile, in 1999, the European Union [1], issued the so-called "Landfill Directive" (1999/31/EC), obliging the Member States to progressively reduce the landfilling of biodegradable waste (European Union, 1999). The "Waste Directive" (2008/98/EC) reinforces the clear intentions of European countries towards a sustainable management of MSW by giving priority (in order of importance) to waste prevention, re-use, recycling, energy recovery and landfilling [2][5]. To achieve these targets, mechanical-biological treatments (MBTs) of MSW have been largely implemented in the last decades [6], with the support of growing efficiencies in the selective collection of waste in industrialised countries [7]. MBTs act on different input waste, such as residual MSW (in the cases of bio drying and biostabilisation), the organic fraction of MSW (OFMSW), green waste and sewage sludge (in the cases of anaerobic digestion and composting). Depending on the process, it is possible to achieve two main different products: bio drying and biostabilisation eliminate or stabilise the putrescible fraction to generate refuse-derived fuel and, in the case of biostabilisation, stabilised material usable as a cover for landfill; anaerobic digestion (AD) and composting produce compost, which, depending on the waste purity, may be used in agriculture. All the presented MBTs respond to the prescriptions of both Directives 1999/31/EC and 2008/98/EC, since these processes reduce the flow of biodegradable waste to landfill and allow for material recycling and energy recovery. The composting of OFMSW is carried out under aerobic conditions, with the addition of green waste as a structuring material, in order to ensure adequate waste porosity and allow for uniform aeration. Since the input waste is a fraction of selective collection, this process leads to material recycling through the production of high-quality compost. On the other hand, $\mathrm{AD}$ allows for energy recovery through the formation of biogas. The latter can be locally exploited by a combined heat and power (CHP) generator, to cover the thermal energy requirements for $\mathrm{AD}$ and produce electric energy for distribution within the electricity network. If the input waste is OFMSW, AD followed by composting of the digestate represents a convenient approach allowing for both energy recovery and material recycling in the same facility [8]. This paper intends to highlight the advantages of modifying 
a hypothetical pre-existing composting plant to an $\mathrm{AD}$ and post-composting plant, in terms of space required and energy balance. The results will be discussed from a qualitative and, whenever possible, quantitative point of view.

\section{CASE STUDY}

As a case study, a hypothetical composting plant is considered as the starting point of this work. A flow diagram of a typical composting facility is presented in Fig. 1(a). The incoming OFMSW is initially discharged in an accumulation chamber, where it is weighed, grinded, sieved and mixed with green waste, which generally accounts for about $30 \%$ of the total waste mass. The mixed waste is sent to a second compartment where it is subject to strong conditions of aerobic biodegradation (biostabilisation) by forced ventilation. This first biological step lasts about 30 days. Subsequently, the stabilised waste mixture is sent to a third compartment (second biological step) where it is subject to natural ventilation allowing for the maturation of compost. The duration of this phase depends on the achieved values of the static and dynamic respirometric indexes, which quantify the level of stability of the treated waste., but it normally lasts for about 60 days. After this period, the compost is sent to a last compartment, where it is stored, packed and prepared for sale. In summary, from its arrival to its storage as compost, a unit of waste spends about 90 days (about 11 weeks) in the composting facility.

The duration of the treatment could be reduced when introducing an AD step upstream of the former biostabilisation step (Fig. 1(b)). This phase takes place in a digester, where the only OFMSW is biodegraded under anaerobic conditions through four phases: hydrolysis (decomposition of organic molecules in simpler ones), acidogenesis (formation of fatty acids, ammonia, carbon dioxide and hydrogen sulphide), acetogenesis (fermentation with formation of carbon dioxide, hydrogen and acetic acid) and methanogenesis (formation of methane, water and carbon dioxide). $\mathrm{AD}$ can be carried out under thermophilic (about $55^{\circ} \mathrm{C}$ ) or mesophilic conditions (about $35^{\circ} \mathrm{C}$ ). The operating temperature has effects on the duration of this phase, ranging (in the case of a semi-dry technology) from about four weeks, under mesophilic conditions, to about 20 days, under thermophilic conditions [9]. During AD, a reduction of the volatile solid (VS) content by about $50 \%$ is typically achieved [10]. The produced digestate generally has a dynamic respirometric index lower than $1000 \mathrm{mg}_{\mathrm{O} 2}$ $\mathrm{kg}_{\mathrm{vs}}{ }^{1} \mathrm{~h}^{-1}$. This allows for a reduced duration of the subsequent aerobic phases (biostabilisation and maturation), which can be contained in a total of about four weeks [11]. Consequently, modifying a classic composting facility to include an AD step may reduce the duration of the whole process by five to six weeks (i.e. by approximately $38-47 \%$ ), depending on the chosen thermal conditions of the AD phase (Table 1).

By the information on the typical duration of each phase and by making realistic assumptions on the typical volumes of the compartments that are necessary for processing the incoming OFMSW, it is possible to compare the surface requirements of each compartment in the hypothetical composting facility and in the modified configuration (AD and post-composting).

A medium-size composting plant is able to treat about 50,000 t/y of waste, typically composed of $70 \%$ OFMSW and $30 \%$ green waste by weight, corresponding to mass flows of $35,000 \mathrm{t} / \mathrm{y}$ and 15,000 t/y of OFMSW and green waste, respectively. Considered the typical densities of compacted food $\left(1,029 \mathrm{~kg} / \mathrm{m}^{3}\right)$ and green waste $\left(445 \mathrm{~kg} / \mathrm{m}^{3}\right)$ reported by the Environmental Protection Authority of the State Government of Victoria (EPA Victoria), the density of the $70 \%-30 \%$ mixture results as about $730 \mathrm{~kg} / \mathrm{m}^{3}$ [12]. The mixture of OFMSW and green waste is often arranged in parallel heaps or trenches, whose top may reach $2 \mathrm{~m}$. Each trench can be approximated as a trapezoidal prism with top base equal to the height 
( $2 \mathrm{~m}$ ) and bottom base of $6 \mathrm{~m}$, by assuming lateral slopes of $45^{\circ}$ (Fig. 2). In order to let operators and forklifts move, each trench can be assumed as surrounded by $2 \mathrm{~m}$ of free space on all sides. The same configuration may be adopted in both biostabilisation and maturation phases, regardless of the considered process (composting or AD and post-composting).

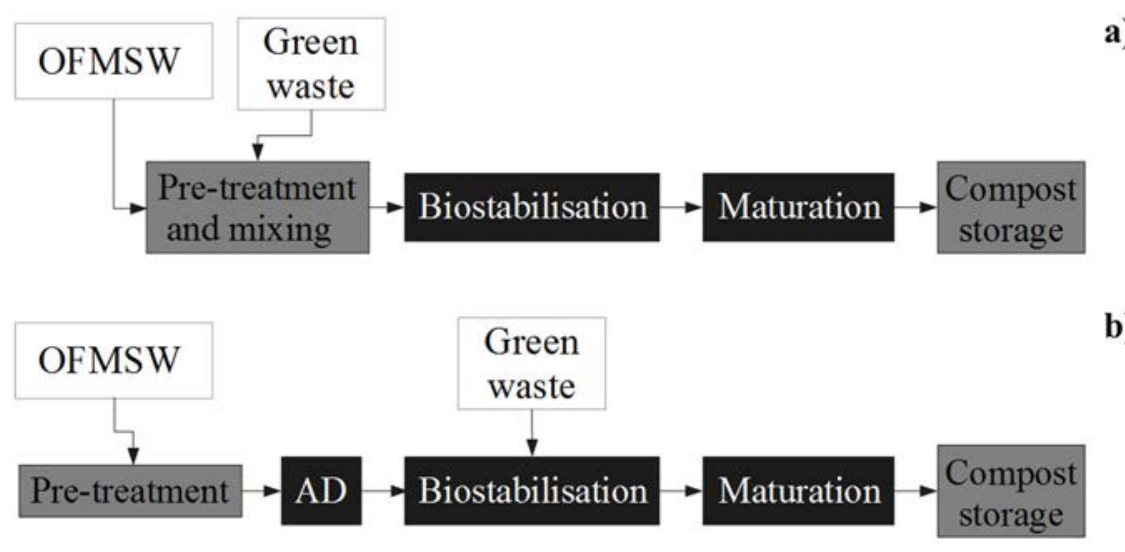

Figure 1: Flow diagrams of (a) the hypothetical composting plant and (b) its conversion to an $\mathrm{AD}$ and post-composting plant.

Table 1: Typical duration (in days) of the phases in the three here considered scenarios.

\begin{tabular}{lccc}
\hline \multicolumn{1}{c}{ Phase } & $\begin{array}{c}\text { Direct } \\
\text { composting }\end{array}$ & $\begin{array}{c}\text { Mesophilic AD and post- } \\
\text { composting }\end{array}$ & $\begin{array}{c}\text { Thermophilic AD and post- } \\
\text { composting }\end{array}$ \\
\hline AD & - & 28 & 20 \\
$\begin{array}{l}\text { Bio- } \\
\text { stabilisation }\end{array}$ & 30 & 14 & 14 \\
Maturation & 60 & 14 & 14 \\
\hline TOTAL & 90 & 56 & 48 \\
\hline
\end{tabular}

Z

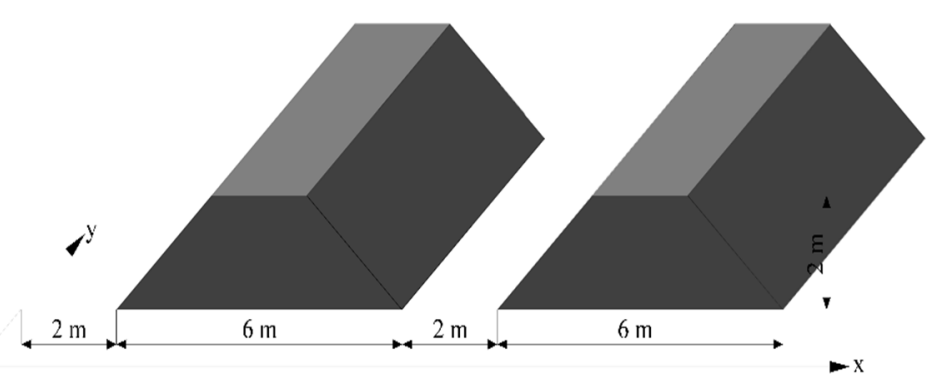

Figure 2: Scheme of the geometric approximation adopted for waste trenches to estimate the surface occupied by the aerobic biodegradation phases. 
In the case of $\mathrm{AD}$ and post-composting, the same mass flow of OFMSW considered in the composting process can be assumed $(35,000 t / y)$. Differently from composting, a lower amount of green waste is usually required in the post-composting process, so that the composition of the input waste is usually $80 \%$ OFMSW (which undergoes AD) and $20 \%$ green waste (which enters the process in the biostabilisation phase). The resulting green waste mass flow is $8,750 \mathrm{t} / \mathrm{y}$. Considered that: 1) the total solid (TS) content and the VS to TS ratio of food waste can be respectively assumed as $70 \%$ and $83 \%$ [13], and 2) AD reduces the VS content by $50 \%$, the digestate mass flow would result as about $25,000 \mathrm{t} / \mathrm{y}$. The digestate density can be assumed as $990 \mathrm{~kg} / \mathrm{m}^{3}$ [14]. By adding the green waste mass flow, a total mass flow of about $34,000 \mathrm{t} / \mathrm{y}$ would undergo the aerobic phases. The mean density of the mixture of digestate and green waste can be estimated as equal to $850 \mathrm{~kg} / \mathrm{m}^{3}$. For simplicity, all calculations consider that the plant operates continuously all year.

\section{RESULTS AND DISCUSSION}

\subsection{Energy requirements}

The introduction of an $\mathrm{AD}$ step into the conventional composting process allows for obvious benefits from the point of view of the energy balance, since AD generates biogas that can be burned in a combined heat and power (CHP) generator. In case AD is carried out under thermophilic conditions, the self-consumption of thermal energy can be assumed as $25 \%$ of the thermal energy produced by the CHP generator. The self-consumption of electric energy is mainly related to the aeration of the heaps in the aerobic phases and can be assumed as $10 \%$ of the electric energy produced by the CHP generator. In the case of direct composting, no thermal energy is required, while electric energy consumption for aeration can be calculated by scaling up the estimation made in the case of $\mathrm{AD}$ and post-composting, according to the higher amount of waste mass flow. The typical productivity of biogas in a semi-dry anaerobic digester is about $100-150 \mathrm{Nm}^{3} / \mathrm{t}_{\mathrm{OFMSW}}$. If considering an average biogas productivity of $125 \mathrm{Nm}^{3} / \mathrm{t}_{\mathrm{OFMSW}}$, a methane content of $60 \%$ in the biogas, a lower heating value of $35.28 \mathrm{MJ} / \mathrm{Nm}^{3}$, thermal and electric energy conversion efficiencies of $40 \%$ and $38 \%$, respectively, both the energy consumptions and productions can be easily estimated (Table 2). In the case of mesophilic AD, the thermal energy required to heat the digester can be estimated with the hypothesis that the mean outdoor temperature is $15^{\circ} \mathrm{C}$ throughout the year. Thus, since the desirable digester temperatures in mesophilic and thermophilic conditions are $35^{\circ} \mathrm{C}$ and $55^{\circ} \mathrm{C}$, respectively, the differences between the target temperatures and the mean outdoor temperature are respectively $20^{\circ} \mathrm{C}$ and $40^{\circ} \mathrm{C}$. By assuming the steadystate relationships for thermal conduction and convective heat transfer, the necessary heat flux is directly proportional to the temperature difference. Consequently, mesophilic conditions would require half of the thermal energy that should be supplied to the digester under thermophilic conditions.

Table 2: Estimated energy balance in the three considered scenarios. Legend: $\mathrm{C}=$ Consumption; $\mathrm{P}=$ Production; $\mathrm{B}=$ Balance, $\mathrm{DC}=$ direct composting; $\mathrm{mAD}, \mathrm{tAD}$ $=$ mesophilic or thermoph. anaerobic digestion.

\begin{tabular}{|c|c|c|c|c|c|c|}
\hline & \multicolumn{3}{|c|}{ Thermal energy $\left[\mathrm{MWh}_{\mathrm{t}} / \mathrm{y}\right]$} & \multicolumn{3}{|c|}{ Electric energy $\left[\mathrm{MWh}_{\mathrm{el}} / \mathrm{y}\right]$} \\
\hline & $\mathrm{C}$ & $\mathrm{P}$ & B & $\mathrm{C}$ & $\mathrm{P}$ & B \\
\hline DC & 0 & 0 & 0 & 1,455 & 0 & $-1,455$ \\
\hline $\mathrm{mAD}$ & 1,286 & 10,290 & 9,004 & 978 & 9,776 & 8,798 \\
\hline tAD & 2,573 & 10,290 & 7,718 & 978 & 9,776 & 8,798 \\
\hline
\end{tabular}


It is clear that direct composting implies a negative energy balance, since electric energy consumption is not compensated by in-situ electric energy production. As expected, mesophilic AD allows for a higher thermal energy balance, since lower energy is necessary to heat the digester. However, even in the case of thermophilic conditions, the biogas exploitation in a CHP generator produces more thermal energy than that required to heat the digester. Thus, a medium-size AD and post-composting facility would be energetically autonomous.

\subsection{Space requirements}

In the case of direct composting, biostabilisation and maturation are supposed to last 30 and 60 days, respectively. The assumed total waste mass flow is $50,000 \mathrm{t} / \mathrm{y}$. Consequently, the biostabilisation and maturation compartments can host about 4,100 t and 8,200 t of waste, respectively, corresponding to volumes of about $5,600 \mathrm{~m}^{3}$ and $11,200 \mathrm{~m}^{3}$. If adopting the trench configuration presented in Section 2 and considering each trench $80-\mathrm{m}$ long, the cross size of the biostabilisation building would measure about $74 \mathrm{~m}$, resulting in an area of 5,920 $\mathrm{m}^{2}$. The maturation building would measure $80 \mathrm{~m} \times 146 \mathrm{~m}$, giving an area of 11,680 $\mathrm{m}^{2} . \mathrm{In}$ total, the biostabilisation and maturation buildings would occupy a surface of $17,600 \mathrm{~m}^{2}$.

In the case of $\mathrm{AD}$ and post-composting, biostabilisation and maturation would last about 14 days each. According to the assumptions made in Section 2, the total waste mass flow would be $34,000 \mathrm{t} / \mathrm{y}$, with mean density of $850 \mathrm{~kg} / \mathrm{m}^{3}$. Therefore, biostabilisation and maturation compartments should host about $1,535 \mathrm{~m}^{3}$ each. If considering the same configurations assumed for the trenches in the case of direct composting, the biostabilisation and maturation buildings would occupy a total surface of $4,800 \mathrm{~m}^{2}$. This surface adds up to that required for the anaerobic digester and the biogas exploitation section. Based on a design parameter retrieved by real plants, the volume required by $\mathrm{AD}$ may be assumed as 0.75 $\mathrm{m}^{3} / \mathrm{t}_{\text {OFMSW }}$. Considered the input OFMSW mass flow of 35,000 t/y and the duration of AD in the case of mesophilic conditions (four weeks), the anaerobic digester would require a volume of about $2,000 \mathrm{~m}^{3}$. If assuming that the anaerobic digester is $5-\mathrm{m}$ high, the occupied surface would be approximately $400 \mathrm{~m}^{2}$. Lower surface would be required if $\mathrm{AD}$ was carried out under thermophilic conditions: due to the lower residence time in the digester (about 20 days, compared to the four weeks required under mesophilic conditions), the resulting surface would be $290 \mathrm{~m}^{2}$. With the exclusion of the space required for the CHP generator, mesophilic and thermophilic $\mathrm{AD}$, followed by post-composting, would require $70 \%$ and $71 \%$ less surface in comparison with the conventional direct composting process, respectively. Fig. 3 presents the results of the surface occupancy in the case of direct composting, mesophilic AD and post-composting, and thermophilic $\mathrm{AD}$ and post-composting, based on the input waste mass flows considered for a medium-size facility. The exclusion of the CHP generator from the estimation of the total surface occupancy is motivated by the extreme compactness of such technology. As an example, a CHP generator capable of developing an output electrical power of $2 \mathrm{MW}_{\mathrm{el}}$ (which would be suitable for an input OFMSW flow rate of about 60,000 $\mathrm{t} / \mathrm{y}$ ) would occupy a surface of about $10 \mathrm{~m}^{2}$. Even considering additional surface for a desulphurisation system (which could be assumed as equal to the surface occupied by the generator), the surface occupancy of the whole biogas exploitation system would represent less than $1 \%$ of the total surface covered by the facility. Thus, in terms of space occupancy, the contribution of the biogas exploitation system is negligible. 


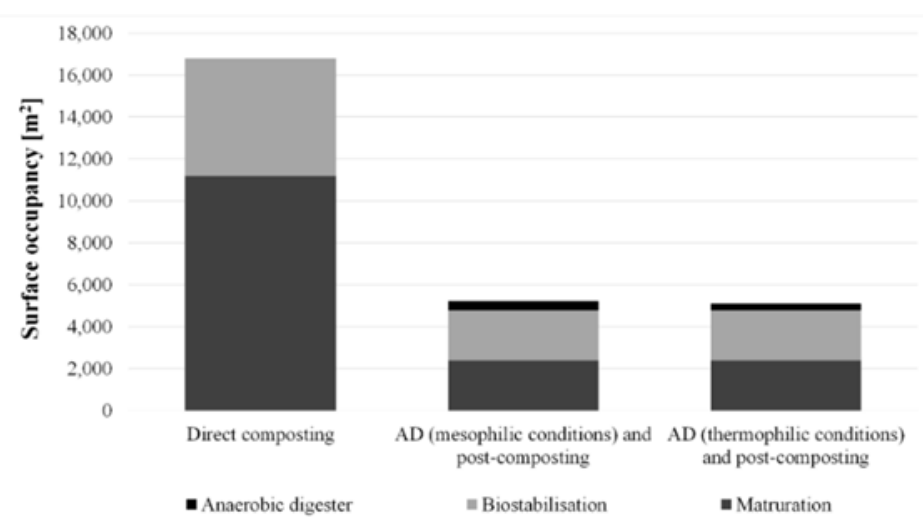

Figure 3: Comparison between the considered scenarios in terms of estimated surface occupancy.

Moreover, additional space would be available by introducing a modification in the air treatment line. Traditionally, the abatement of odours, volatile organic compounds, sulphides and ammonia released in the waste air of MBTs is carried out by the adoption of open-bed biofilters [15]-[20]. Due to the need for ensuring a sufficient residence time for the biodegradation of air pollutants and homogeneity of the treatment, biofilters require large areas to operate properly and to allow for satisfying removal efficiency. Operatively, biofilters must meet specific design criteria [21], concerning the superficial gas velocity (i.e. the outlet velocity of the treated gas normalised to the biofilter area), the volumetric load (i.e. the airflow rate normalised to the biofilter volume) and the empty-bed residence time (i.e. the time requested by the influent gaseous stream to pass through the empty volume that the filtering bed would occupy). Recent studies [22], [23], highlighted the benefits achievable when replacing conventional biofilters with the so-called bio-trickling filters (BTFs). Benefits are also achievable in terms of space reduction, mainly thanks to the generally lower empty-bed residence time required by BTFs and to the less stringent requirements on the superficial gas velocity, which make BTFs more compact and developed in the vertical dimension. According to a recent work [24], the replacement of a biofilter with an equivalent air treatment system based on BTFs would reduce the area for air treatment by about $85 \%$, value significant in the frame of waste treatment plant enhancement [25].

\section{CONCLUSIONS}

The contemporary recovery of material and energy achievable when integrating AD with composting perfectly interprets the hierarchy of priorities given by the current environmental legislation. Indeed, in an exclusively aerobic process, volatile organic matter would be oxidised to $\mathrm{CO} 2$ with no energy recovery. On the other hand, the addition of an anaerobic step allows both transforming volatile organic matter in a substance (biogas) that is exploitable for energy purposes and preserving the agronomic value of the remaining amount of organic carbon, which is transformed into compost. From the point of view of the energy balance, this conversion is surely positive. The excess energy produced in the AD stage could be sold to the market: electric energy could be distributed through the electricity network and thermal energy could be supplied to the local district heating. In addition, the introduction of 
the anaerobic stage allow reducing the space occupied by the former composting facility for three main reasons:

- the residence time of the input waste in the facility may be up to $47 \%$ lower than direct composting, and lower residence time entails lower compartment volumes;

- the anaerobic pre-treatment reduces the waste mass flow entering the aerobic compartments, which are the most surface-consuming stages of the process;

- $\mathrm{AD}$ and biogas exploitation systems are considerably compact technologies with respect to the volume required by aerobic biodegradation.

As a result, in the case of a typical medium-size plant, a $70 \%$ reduction in the surface occupancy might be achieved when converting a direct composting plant into an AD and post-composting process. Such a choice would reduce the investment costs to purchase terrains and build the aerobic compartments. On the other hand, the initial investment would necessary include the purchase of the anaerobic digester and the biogas exploitation system. However, the possibility to sell the excess produced energy would ensure an economic return covering the initial investments in the midterm and additional long-term profit. The choice of operating $\mathrm{AD}$ under mesophilic or thermophilic conditions seems not to have particular influence on the space required by a medium-size facility. From the point of view of the energy balance, if supplying the net thermal energy to the local district heating network were an option, mesophilic conditions would be preferable, since a higher amount of net thermal energy would be available.

\section{REFERENCES}

[1] European Union, Council Directive 1999/31/EC of 26 April 1999 on the landfill of waste. Official Journal of the European Union L 182, pp. 1-19, 1999.

[2] European Union, Directive 2008/98/EC of the European Parliament and of the Council of 19 November 2008 on waste and repealing certain Directives. Official Journal of the European Union L 312(3), pp. 3-30, 2008.

[3] Rada, E.C., Present and future of SRF. Waste Management, 47, pp. 155-156, 2016.

[4] Ionescu, G., Rada, E.C. \& Cioca, L.I., Municipal solid waste sorting and treatment schemes for the maximization of material and energy recovery in a latest EU member. Environmental Engineering and Management Journal, 14(11), pp. 2537-2544, 2015.

[5] Rada, E.C., Energy from municipal solid waste. WIT Transactions on Ecology and the Environment, 190(2), pp. 945-958, 2014.

[6] Ragazzi, M., Tosi, P., Rada, E.C., Torretta, V. \& Schiavon, M., Effluents from MBT plants: Plasma techniques for the treatment of VOCs. Waste Management, 34(11), pp. 2400-2406, 2014.

[7] Rada, E.C., Bresciani, C., Girelli, E., Ragazzi, M., Schiavon, M. \& Torretta, V., Analysis and measures to improve waste management in schools. Sustainability, 8, p. 80, 2016.

[8] Smet, E., Van Langenhove, H. \& De Bo, I., The emission of volatile compounds during the aerobic and he combined anaerobic/aerobic composting of biowaste. Atmospheric Environment 33(8), pp. 1295-1303, 1999.

[9] Italian Composting Consortium, L'integrazione tra la digestione anaerobica e il compostaggio (The integration between anaerobic digestion and composting). Technical report (in Italian), 2006.

[10] Haug, R.T., Substrate Biodegradability. Lewis Publishers, Boca Raton, p. 752, 1993. 
[11] Spuhler, Anaerobic Digestion (Organic Waste). Sustainable Sanitation and Water Management. Available at: http://www.sswm.info/content/anaerobic-digestionorganic-waste, 2017.

[12] EPA Victoria, Waste Materials - Density Data. Environmental Protection Authority of the State Government of Victoria. Available at: http://www.epa.vic.gov.au/businessand-industry/lower-your-impact/ /media/Files/bus/EREP/docs/wastematerialsdensities-data.pdf, 2017

[13] Zhang, R., El-Mashad, H.M., Hartman, K., Wang, F., Liu, G., Choate, C. \& Gamble, P., Characterization of food waste as feedstock for anaerobic digestion. Bioresource Technology, 98, pp. 929-935, 2007.

[14] Waste and Resources Action Programme, Digestates: Realising the fertilizer benefits for crops and grassland. Technical report No. OAV036-210, 2011.

[15] Clemens, J. \& Cuhls, C., Greenhouse gas emissions from mechanical and biological waste treatment of municipal waste. Environmental Technology, 24, pp. 745-754, 2003.

[16] Velis, C.A., Longhurst, P.J., Drew, G.H., Smith, R. \& Pollard, S.J.T., Biodrying for mechanical-biological treatment of wastes: A review of process science and engineering. Bioresource Technology, 100(11), pp. 2747-2761, 2009.

[17] Cabrera, G., Ramírez, M. \& Cantero, D., Biofilters. In: Moo-Young, M. (ed.), Comprehensive Biotechnology, Academic Press, Burlington, pp. 303-318, 2011.

[18] Schiavon, M., Scapinello, M., Tosi, P., Ragazzi, M., Torretta, V. \& Rada, E.C., Potential of non-thermal plasmas for helping the biodegradation of volatile organic compounds (VOCs) released by waste management plants. Journal of Cleaner Production, 104, pp. 211-219, 2015.

[19] Torretta, V., Collivignarelli, M.C., Raboni, M. \& Viotti, P., Experimental treatment of a refinery waste air stream, for BTEX removal, by water scrubbing and biotrickling on a bed of Mitilus edulis shells. Environmental Technology, 36, pp. 2300-2307, 2015.

[20] Charles, W. \& Ho, G., Biological Methods of Odor Removal in Solid Waste Treatment Facilities. In: Wong, J.W.C., Tyagi, R.D. \& Pandey, A. (eds.), Current Developments in Biotechnology and Bioengineering, Elsevier, Amsterdam, pp. 341-365, 2017.

[21] Lebrero, R, Muñoz, R, Villaverde, S., Hung, Y.-T., Air Biofiltration Applied to Odor Treatment. In: Hung, Y.-T., Wang, L.K. \& Shammas, N.K. (eds.), Handbook of Environment and Waste Management. World Scientific Publishing, Singapore, pp. 149-174, 2012.

[22] Álvarez-Hornos, F.J., Lafita, C., Martínez-Soria, V., Penya-Roja, J.M., Pérez, M.C. \& Gabaldón, C., Evaluation of a pilot-scale biotrickling filter as a VOC control technology for the plastic coating sector. Biochemical Engineering Journal, 58, 59, pp. 154-161, 2011.

[23] Copelli, S., Torretta, V., Raboni, M., Viotti, P., Luciano, A., Mancini, G. \& Nano, G., Improving biotreatment efficiency of hot waste air streams: Experimental upgrade of a full plant. Chemical Engineering Transactions, 30, pp. 49-54, 2012.

[24] Schiavon, M., Ragazzi, M., Torretta, V. \& Rada, E.C., Comparison between conventional biofilters and biotrickling filters applied to waste bio-drying in terms of atmospheric dispersion and air quality. Environmental Technology, 37(8), pp. 975982, 2016.

[25] Ionescu, G., Rada, E.C., Ragazzi, M., Mărculescu, C., Badea, A. \& Apostol, T., Integrated municipal solid waste scenario model using advanced pre-treatment and waste to energy processes. Energy Conversion and Management, 76, pp. 1083-1092, 2013. 